\title{
À propos de la distinction entre institution et organisation chez les institutionnalises : apports et limites
}

\section{Christian Palloix}

\section{(2) OpenEdition Journals \\ Édition électronique \\ URL : http://journals.openedition.org/ei/708 \\ DOI : $10.4000 /$ ei. 708 \\ ISSN : 2553-1891 \\ Éditeur \\ Association Économie et Institutions}

\section{Édition imprimée}

Date de publication : 1 décembre 2002

Pagination : 67-98

ISSN : 1775-2329

\section{Référence électronique}

Christian Palloix, « À propos de la distinction entre institution et organisation chez les

institutionnalises : apports et limites », Économie et institutions [En ligne], 1 | 2002, mis en ligne le 31 janvier 2013, consulté le 23 avril 2019. URL : http://journals.openedition.org/ei/708; DOI : 10.4000/ ei. 708 


\title{
A propose de la distinction entre institution et organisation chez les institutionnalises : apports et limites *
}

\author{
Christian PALLOIX \\ C.R.I.I.S.E.A. \\ UPJV - AMIENS
}

"L'Efficiency - la Performance - est le nom nouveau qui donne figure humaine à l'Abîme. La marche technologique balaye les faibles, comme les guerres autrefois: elle réinvente le sacrifice humain de façon douce ; elle fait régner l'harmonie par le calcul."

Pierre Legendre, La fabrique de l'homme occidental, Editions Mille et une nuits/Arte, 1996, p.26

L'institutionnalisme est traditionnellement présenté sur la base de deux grands courants de pensée, "the old institutionalism " (O.I.), avec pour principaux acteurs T.Veblen et J.R.Commons, et "the new institutionalism " couramment dénommé la nouvelle économie institutionnaliste (N.E.I.), avec de très nombreux auteurs, dont notamment R.H.Coase, R.N.Langlois, D.C.North, O.E.Williamson, ....tout en rappelant que ce dernier courant se subdivise en plusieurs sous-courants ${ }^{1}$.

L'institutionnalisme globalement, même si certains récusent toute filiation de pensée entre la N.E.I. et l'O.I., paraît traversé par une double question :

- quelle distinction entre institution et organisation?

- l'organisation est-elle une construction endogène à l'institution ou une construction exogène qui lui est juxtaposée?

Cette double question alimente l'institutionnalisme qui se développe apparemment sur deux grands courants de pensée opposés, l'un l'O.I. affilié à l'économie politique classique, l'autre la N.E.I. à l'école néoclassique. En effet ces questions conduisent à la construction d'un dispositif commun plus ou moins proche,

\footnotetext{
* Cet article est une version révisée d'une communication au colloque "Institutions et Organisations", 25-26 mai 2000, Amiens. Je tiens à remercier les différents rapporteurs anonymes pour leurs remarques et suggestions relatives à la première version proposée pour publication.

${ }^{1}$ On ne tiendra pas compte ici du courant évolutionniste (Dosi, Nelson, Winter) qui nous paraît camper davantage sur le champ de l'organisation.
} 
propulsant l'institutionnalisme globalement comme une école un peu à part, avec ses ouvertures face aux fermetures, standard,

- et de l'économie néoclassique également dénommée économie standard.

et de l'économie classique appelée aussi économie non

Comme le soutient G.Hodgson [2000], l'approche néoclassique ou économie standard se caractérise par trois traits essentiels :

"1) elle suppose une conduite rationnelle et maximisatrice des agents dotés de fonctions de préférence données et stables,

2) elle met l'accent sur des situations d'équilibre, soit atteintes ou en cours, (p.29),

3) elle est marquée par l'absence de tout problème d'information"

ce qui exclut l'école autrichienne (Hayek, Von Mises notamment) de l'école néoclassique selon Hodgson quant à un possible ralliement aux points 2 et 3 .

Par économie classique ou économie non standard, on désigne le courant de pensée qui court d'Adam Smith (à la croisée des chemins classique et néoclassique) et D.Ricardo à K.Marx, J.M.Keynes, P.Sraffa et autres, et qui a pour principale caractéristique d'ancrer les modalités de l'échange des biens et services (entre les agents) dans les conditions de production et de répartition, relâchant quelque peu (avec de fortes différences d'un sous-courant à l'autre) l'importance du comportement des agents.

Dans un premier temps sera proposé le contenu d'un dispositif institutionnaliste - sur institution et organisation - qui nous paraît au cœur de l'analyse de l'O.I. et de la N.E.I., dispositif plus complet dans l'O.I., plus tronqué dans la N.E.I. Ce dispositif fonctionne comme mode de lecture et de questionnement des différents auteurs. Ainsi, dans un deuxième temps, ce dispositif sera décliné par rapport à l'O.I. et plus particulièrement Veblen et Commons, avec leurs points communs, leurs différences. Ce dispositif est appliqué ensuite sur les principaux auteurs de la N.E.I.

\section{Originalité et ambiguïté du dispositif institutionnaliste}

Je propose de rassembler comme suit les différentes combinaisons à l'œuvre au sein du dispositif institutionnaliste,

- tant de l'O.I. (T.Veblen, J.R.Commons), 
- que de la N.E.I. (R.H.Coase, R.N.Langlois, D.C.North, O.E.Williamson).

Signalons qu'il n'en demeure pas moins des oppositions plus ou moins fortes entre l'O.I. et la N.E.I., notamment au niveau de l'analyse du comportement des individus, comme le soutiennent d'un côté G.Hodgson [1998] et de l'autre R.N.Langlois [1990]² en campant sur des positions opposées.

Première combinaison (C1) : L'économie institutionnaliste propose un arrangement particulier de l'économie, ou arrangement institutionnel.

L'arrangement institutionnel ${ }^{3}$ - qui n'est nullement pris ici comme "structure de gouvernance" à la manière d'O.E.Williamson s'appuie sur une trilogie privilégiée des composantes de l'économie (marchande et monétaire) avec

- comportements des agents,

- actifs,

- institutions, comme régularités de comportements,

où les institutions assurent tout à la fois coordination et combinaison entre les deux composantes de l'économie, les comportements des individus d'un côté et les actifs de l'économie de l'autre ${ }^{4}$, sur la base du recours aux droits de propriété.

Nous ne sommes pas ici sur la boucle institutionnaliste néoclassique traditionnelle rappelée par G.Hodgson selon la figure 1 ci-après.

\footnotetext{
${ }^{2}$ R.N.Langlois [1990] : "L'école institutionnaliste américaine était un groupe éclaté et éclectique... De fait, il était si faiblement structuré comme groupe que le dénominateur commun parmi ses membres était peut-être leur opposition au point de vue néoclassique qui se développait alors "(pp.2-3). La disqualification de Veblen et de Commons par R.N.Langlois porte sur le fondement théorique de l'analyse des comportements des individus, notamment chez Veblen (p. 4)

3 Le concept d'arrangement institutionnel doit être entendu ici comme différent de celui d'arrangement institutionnel de L.E.Davis et D.C.North [1971] avec un arrangement institutionnel stricto sensu et un environnement institutionnel, distinction opérée par et rappelée par P.G.Klein [1998].

${ }^{4} \mathrm{Si}$ ces composantes ne sont pas celles de l'économie non standard, qui se lie plus particulièrement à marchandise (et monnaie), capital, salariat, structures - cf. la présentation canonique par P. Sraffa [1960] "d'une production de marchandises par des marchandises "-, les deux dispositifs ont une certaine proximité relative si " actif " est rapproché de " capital ", et "institution " de " structure ".
} 
Figure 1. La boucle institutionnaliste traditionnelle "Action-Information"

(Schéma emprunté à G.Hodgson [1998])

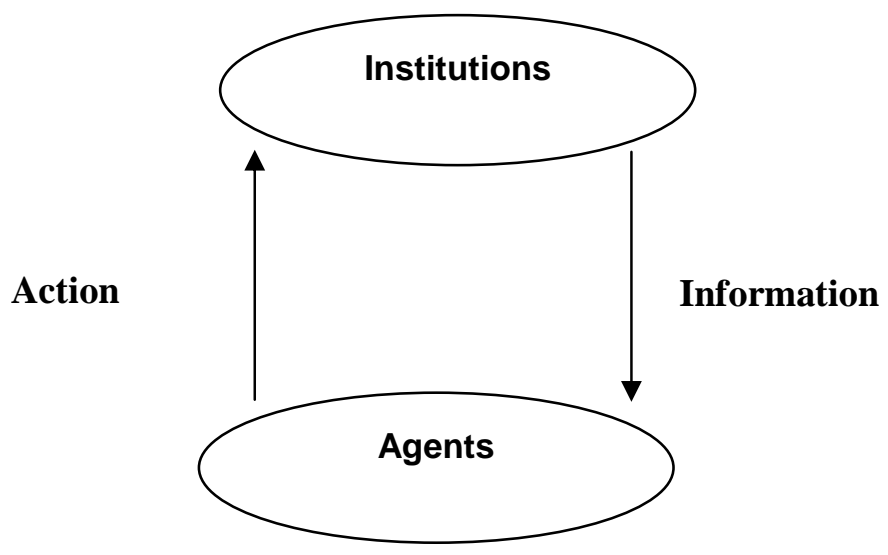

Nous sommes sur plusieurs boucles selon la figure 2, où les institutions ont pour enjeu le comportement des individus (sur la base d'une rationalité procédurale pour le moins ) quant aux actifs à disposition et mobilisables (droits de propriété, efficience, modalités de répartition des richesses produites et reproduites par les actifs, .....)

Figure 2 - La combinaison de l'arrangement institutionnel "Agents, Actifs, Institutions"




Les institutions représentent les différents arrangements (avec leurs règles, habitudes) d'un couple agents-actifs qui court du marché à la firme, leur mise en forme selon des déclinaisons multiples qui tiennent à la diversité des agents, à la diversité des actifs, à la diversité des situations et des environnements, .... Agents et actifs se distribuent, rentrent dans des arrangements institutionnels spécifiques. Les institutions mettent en route le fonctionnement de l'économie (agents, actifs), et assurent la coordination globale sur le plan de la valorisation des actifs.

Certes, tant dans la première approche institutionnaliste de T.Veblen et de J.R.Commons que dans celle de R.H.Coase, R.N.Langlois, D.C.North, O.E.Williamson, les représentations de l'économie diffèrent, et en appellent à d'autres composantes,

- soit alternatives (transaction à la place d'actif chez J.R.Commons),

- soit complémentaires (transactions, contrats, ..., dans la N.E.I.).

Dans ce contexte, l'arrangement institutionnel se décline selon une pluralité de modes (des prix au réseau) de coordination (dynamique, interne, externe) des agents (individus et institutions elles-mêmes) dans une logique de valorisation.

\section{Deuxième combinaison (C2) : L'arrangement institutionnel se donne un arrangement organisationnel.}

La première combinaison est incomplète. Les institutions, qui distribuent leurs rôles aux agents et aux actifs en économie de marché et d'entreprise, sont des boîtes encore vides (un "cadre " comme l'assument nombre d'auteurs institutionnalistes) sans une organisation des agents et des actifs qui les transcende et les dépasse ${ }^{5}$. Ce sont des boîtes qui ne se sont pas encore emplies de l'acte technologique, productif, commercial, créateur de surplus (ou richesses) et opérateur de transfert de surplus (ou richesses).

L'économie institutionnaliste, tant l'O.I. que la N.E.I., est alors contrainte de produire une analyse jointe de l'organisation, un arrangement organisationnel. Cet arrangement organisationnel (C2) se déploie lui aussi sur trois composantes principales,

- comportements des agents,

- actifs,

- organisations, comme règles de comportements,

\footnotetext{
${ }^{5}$ La boîte institutionnelle est encore vide, à ce moment-là, du contenu que donne l'organisation dans ses différentes appellations selon la diversité des écoles de pensée, force productive chez les marxistes par exemple, efficience en économie standard.
} 
où les organisations (au lieu et place d'institutions comme dans $\mathrm{C} 1$ ) représentent différents arrangements efficients portant sur les comportements des individus d'un côté et les actifs de l'économie de l'autre, sur la base du recours à la hiérarchie (au lieu et place de droits de propriété comme dans $\mathrm{C} 1$ ). L'organisation remplace l'institution dans les boucles de la figure 3 ci-après.

\section{Figure 3 - La combinaison de l'arrangement organisationnel} "Agents, Actifs, Organisations"

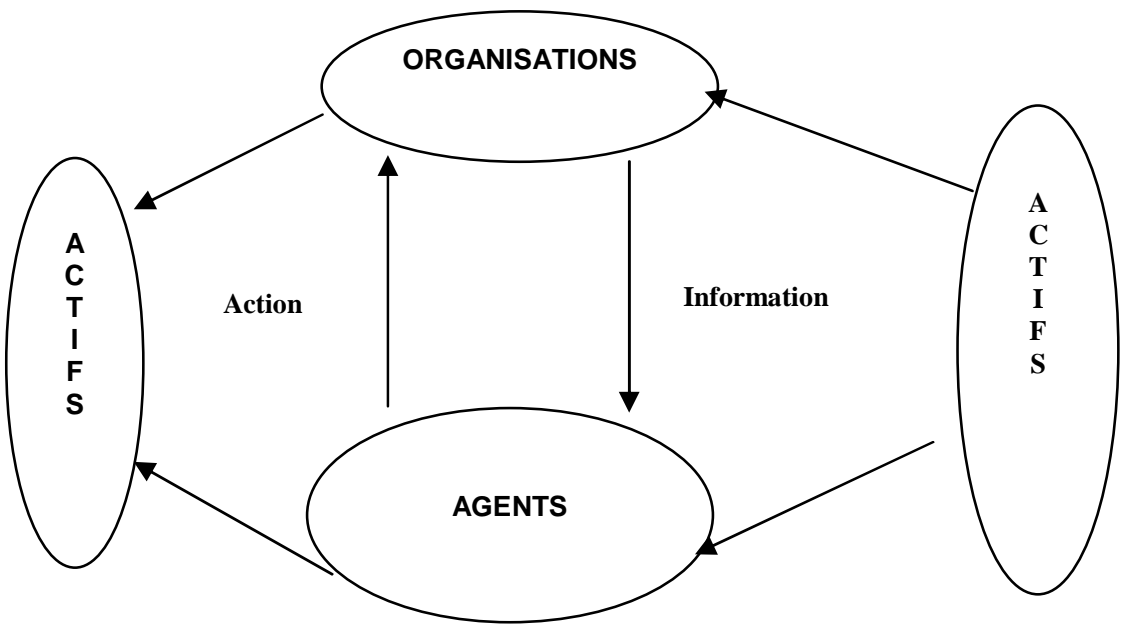

La clef de passage entre "institution " (C1) et " organisation " (C2) paraît se jouer au niveau des "règles ", comme l'expose B.Chavance [1998], avec une distinction entre les règles institutionnelles et les règles organisationnelles, cette coupure renvoyant à droits de propriété d'un côté et hiérarchie de l'autre.

La distinction de l'arrangement institutionnel et de l'arrangement organisationnel conduit soit à leur juxtaposition (O.I. de T.Veblen et N.E.I. "williamsonienne", soit à leur imbrication apparente plus ou moins fusionnelle (O.I. de J.R.Commons, N.E.I. chez D.C. North ou chez R.N.Langlois), avec des couplages pas toujours attendus.

Toute la question est de savoir si l'arrangement institutionnel porte en lui, dans ses fondements et sa logique interne, la genèse organique endogène d'un arrangement organisationnel. L'organisation paraît avoir vocation, dans l'O.I., à fournir la capacité de capture par l'institution d'une puissance productive qui lui demeure extérieure. La validation des institutions ne réside pas 
seulement dans les fondements de l'arrangement institutionnel (coordination des agents), mais aussi dans l'emprunt et l'usage de quelque chose qui leur est extérieur.

Troisième combinaison (C3) : selon que le couplage de l'organisation à l'arrangement institutionnel affiche C2 comme une extériorité (O.I. de Veblen) ou non (O.I. de Commons et N.E.I.), des conflits distributifs traversent ou non l'économie.

Les conflits distributifs, particulièrement forts chez T.Veblen (entre la classe de loisir et la classe ouvrière, entre l'économie financière - le monde des affaires - et l'économie industrielle), sont déjà plus contenus et normalisés chez J.R.Commons. Tout s'enracine dans une théorie des prix, prix entendus comme variables de répartition, plus ou moins maintenue. L'actualité de T.Veblen est dans la mise en évidence du conflit distributif entre le monde des affaires d'un côté et l'économie industrielle de l'autre, conflit particulièrement pertinent pour l'analyse du capitalisme aujourd'hui [Palloix 1996].

La N.E.I. ignore pratiquement tout conflit distributif. Cela paraît tenir pour partie à l'absence d'un enracinement endogène de l'analyse dans une théorie des prix et de la monnaie. L'absence de prise en compte du conflit distributif est aussi à rechercher dans l'effacement d'une théorie de l'organisation qui est rabattue sur l'analyse institutionnelle, institution et organisation se confondant. Dès lors, la question de l'usage d'une force extérieure - et de son affectation - par l'institution ne paraît pas se poser.

\section{La déclinaison spécifique du dispositif fondateur dans "the old institutionalism " (O.I)}

Si T.Veblen et J.R.Commons présentent une forte proximité de leur dispositif d'analyse des institutions et du comportement des individus (le refus de l'individualisme méthodologique, une conception plus holiste qu'individualiste), les divergences pointent quant aux fondements organisationnels et conflits distributifs. 
2.1. Un dispositif relativement commun à Veblen et Commons : Comportements des individus et institutions (C1)

Tant pour T.Veblen que pour J.R.Commons, les institutions naissent des habitudes. Comme le souligne G.Hodgson [1998], trois mots paraissent résumer les fondements de l'O.I., "habitudes, règles, institutions "; l'analyse du comportement de l'individu s'inscrit dans les habitudes, règles, et celles-ci sont connectées à l'analyse des institutions : "L'argument clef de mon article est que le vieil institutionnalisme offre une perspective radicalement différente quant à la nature du comportement humain, qui repose sur le concept d'habitude. ...Dans une optique institutionnaliste, le concept d'habitude est connecté fondamentalement avec l'analyse des institutions " . (1998, p.167)

\subsubsection{L'arrangement institutionnel chez T. Veblen}

Dans une version très dense, T.Veblen livre ci-après les éléments fondamentaux d'une théorie des institutions fondée sur la relation dialectique entre les couples habitudes-normes, et adaptation-sélection, où

- d'un côté, les institutions, nées des habitudes, sont les résultats d'un processus adaptatif et de sélection "des habitudes mentales les plus recevables ", d'évolution et d'adaptation forcées au milieu,

- et de l'autre, elles sont " des méthodes particulières de vie et de relations humaines ", i.e. des règles, et qui s'imposent quant à la coordination des individus et qui favorisent la sélection et l'adaptation des individus à l'évolution :

- "L'évolution de la structure sociale a été un processus de sélection naturelle des institutions. Les institutions humaines ont fait et font encore des progrès qui se réduisent en gros à une sélection naturelle des habitudes mentales les plus recevables, et à un processus d'adaptation forcée des individus à leur milieu, un milieu qui a changé au fur et à mesure que la société se développait et que changeaient aussi les institutions sous lesquelles les hommes ont vécu. Les institutions elles-mêmes ne sont pas seulement les résultats d'un processus sélectif et adaptatif, qui façonne les types prédominants d'attitude et d'aptitude spirituelle; elles sont en même temps des méthodes particulières de vie et de relations humaines, et à ce titre elles sont à leur tour de puissants facteurs de sélection. En sorte que les institutions, quand elles changent, favorisent une nouvelle sélection des individus doués du tempérament le plus 
approprié; elles aident le tempérament et les habitudes à se plier au milieu transformé, grâce à la formation d'institutions nouvelles." (1899, p.124),

- "L'adaptation des façons de penser, c'est le développement même des institutions. "(1899, p.140)

Dans ce processus où les institutions naissent des habitudes ${ }^{6}$, et où les institutions façonnent les habitudes comme régularités de comportements, dans un aller-retour sans fin, il est clair que l'individualisme méthodologique de l'agent sous rationalité substantive - en information parfaite - dans la conduite de "Max U " est disqualifié : il s'y substitue une analyse davantage holiste des comportements.

T. Veblen ouvre dès 1899 une analyse des droits de propriété, dans un processus historique qui court de la propriété des femmes ${ }^{7}$ à celle des biens ${ }^{8}$. Les fondements de la propriété sont la rivalité, évoluant progressivement vers la " rivalité pécuniaire " :

- "Le motif qui se trouve à la racine de la propriété, c'est la rivalité; c'est le même qui continue dans cette institution qu'il a fait naître, et dans le déploiement de tous ces traits de la structure sociale qui touchent à l'institution de la propriété. La possession des richesses confère l'honneur: c'est une distinction provocante. "(1899, p.19).

- Avec l'évolution vers une propriété industrielle, la rivalité devient une rivalité pécuniaire : "Parmi tous les motifs qui poussent l'homme à accumuler des biens, c'est à la rivalité pécuniaire qu'appartient la primauté." (1899, p.25)

Par ailleurs, T.Veblen apporte une distinction entre les institutions pécuniaires et les institutions industrielles, de laquelle dérive - en fonction du droit de propriété - une distinction entre la classe de loisir et la classe laborieuse, la classe étant l'institution première. Les institutions concernent non seulement les rapports entre les individus, mais aussi les rapports entre les individus et le

\footnotetext{
6 " Une institution doit changer selon les circonstances puisqu'elle tient de l'habitude, c'est-à-dire qu'elle est une façon accoutumée de répondre aux stimuli que ces circonstances apportent en changeant. Le développement de ces institutions, c'est le développement de la société. En substance, les institutions sont des habitudes mentales prédominantes, des façons très répandues de penser les rapports particuliers et les fonctions particulières de l'individu et de la société. " (1899, p.125) 7 « On a lieu de croire que l'institution de la propriété a commencé par la propriété des personnes, et tout d'abord des femmes. Pour acquérir ces sortes de biens, les hommes ont été stimulés par $1^{\circ}$ la tendance à dominer et à contraindre ; $2^{\circ}$ 1'utilité de ces personnes comme témoignage de la vaillance de leur possesseur ; $3^{\circ}$ l'utilité de leurs services. " $(1899$, p.37)

8 " Dans le cours de l'évolution culturelle, l'émergence d'une classe oisive coïncide avec les débuts de la propriété...... ces deux institutions résultent d'une même série de forces économiques. " $(1899, \mathrm{p} .17)$
} 
monde matériel: "On peut regarder toute société comme un mécanisme industriel ou économique, dont la structure est l'assemblage des institutions que l'on appelle économiques. Ces institutions sont les méthodes auxquelles on recourt habituellement pour entretenir le processus vital de la société, au contact du milieu matériel. " (1899, p.127).

Avec "processus vital de la société", la question de l'organisation n'est pas loin et n'est pas sans analogie avec "l'action collective " ou "going concern" de J.R.Commons.

Il en découle une séparation des institutions de classe selon la nature de la rivalité, le travail pour les uns, l'argent pour les autres :

- classe laborieuse, "Ces classes inférieures n'ont aucun moyen d'échapper au travail ... Etant donné que le travail est leur mode de vie reconnu et admis, on $y$ est plutôt fier d'avoir la réputation d'un travailleur capable; c'est souvent là toute l'émulation qui leur est permise. "(1899, p.26)

- classe de loisir, "...la vie de loisir est belle et ennoblissante aux yeux de tout homme civilisé..... L'abstention affichée de tout travail devient ... la preuve classique de l'exploit pécuniaire. "(1899, p.28)

Si l'arrangement institutionnel - où les institutions naissent des habitudes et règles - de T.Veblen se présente comme un processus généralisé de coordination, cette coordination est davantage saisie dans une dimension dynamique de sélection et d'adaptation.

2.1.2. L'arrangement institutionnel de J.R.Commons et premières divergences avec T.Veblen

Les premiers textes de J.R.Commons sont forts éclairants sur la construction du dispositif qui vise à résoudre le passage entre la facette " arrangement institutionnel " et la facette " organisation ".

Les fondements initiaux de l'analyse des institutions de J.R.Commons dans ses rapports avec les comportements des individus sont fort proches de ceux de T.Veblen. De plus, les institutions ne portent pas seulement sur la coordination des individus, mais visent, comme chez T.Veblen, la satisfaction des besoins des agents à partir d'une production matérielle.

Mais, tout aussitôt, J.R.Commons introduit de nouveaux éléments à son analyse qui le démarquent de T.Veblen, avec une mise en place de l'institution sous un triple statut: 
- celui de l'unité interne de l'institution, fondée sur l'action collective où l'action apparaît comme le fondement de la coordination interne de l'institution, celle-ci faisant également appel aux droits de propriété,

- celui de la coordination (unité) des institutions (comment tout tient ensemble), avec mise en place d'une coordination externe,

- l'organisation venant supporter le point de vue institutionnel premier, ainsi que cela apparaît dans le texte ci-après : "En conséquence, une double relation de ses membres se développe, premièrement comme relation collective, en liaison avec les autres institutions, et deuxièmement comme relation individuelle, en liaison avec un autre membre. L'unité d'une institution est sa capacité de développer des actions jointes avec d'autres membres et d'autres institutions. Elle agit comme unité; ses membres gomment leurs différences; elle a une volonté commune. Ceci génère énergie et puissance. Les croyances et les désirs communs sont les forces vitales, actives au sein de l'institution. Les produits matériels représentent sa commande sur la nature. L'organisation lui donne son unité et son commandement sur la société. "(1899-1900, p. 66)

Nous voilà au cœur du problème de la démarcation éventuelle entre l'institution et l'organisation : l'organisation surgit, émerge comme action (going concern) de l'institution, l'organisation donnant sens en retour à l'institution.

Un autre élément de différenciation de l'analyse des institutions chez J.R.Commons tient à la prise en compte de la rareté $^{9}$ des ressources, ce qui le conduit à la légitimation de l'efficience du droit de propriété comme forme de gestion de ressources rares : "C'est l'accroissement de la densité de population qui conduit à la prise de conscience de l'élément de rareté dans les divers champs de l'activité humaine l'un après l'autre, et sur cette prise de conscience l'appropriation privée se construit, posant alors la fondation des institutions sociales. Toutes les institutions sociales ont pour origine la propriété privée... la propriété privée est l'expression sociale de l'unité la plus élevée de l'individu, sa prise de conscience." (1899-1900, p. 71-72),

soit une nette prise de distance par rapport à T.Veblen.

Enfin, progressivement, dans le corpus d'analyse des institutions, leur inscription ne repose plus sur l'acte d'échange, mais sur la transaction, avec une différenciation entre transaction

${ }^{9}$ Ceci se retrouve dans l'article de 1932 , où J.R.Commons renvoie à D.Hume sur les fondements de l'analyse de la rareté. 
d'échange, transaction de management (efficience, organisation), transaction de répartition ${ }^{10}$.

De fait, le dispositif de J.R.Commons apparaît beaucoup plus " ramassé " que celui de T.Veblen, les combinaisons C1, C2, C3 se supposant réciproquement dans un tout difficilement séparable : par exemple, la transaction est transaction d'organisation (C2), transaction de répartition (C3), tout en étant au cœur du dispositif premier de l'arrangement institutionnel de l'échange (C1). La distinction des éléments du dispositif global d'analyse est plus délicate à opérer.

\section{Conclusions sur ce point de l'arrangement institutionnel dans 1'O.I. :}

1) On pourrait penser que les fondements institutionnels de l'O.I. dans des habitudes et règles, qui renvoient à une perception relativement holiste de l'individu, représentent une version archaïque et déclassée de l'analyse du comportement des individus. Cette vue est réductrice comme si holisme et individualisme s'excluaient réciproquement. L'O.I. nous livre l'individu sous une double face liée,

- l'individu qui entre dans le collectif et part du collectif par les habitudes et règles (la face holiste qui naît de l'individu séparé),

- et un individu (holiste) qui entre dans un processus d'individuation de sélection et d'adaptation (la face individualiste qui naît de l'individu holiste).

Cette modernité de l'analyse des comportements de l'individu s'exprime notamment dans la relative similitude du concept "d'habitude " (holiste-individualiste) développé dans l'O.I. avec celui "d'habitus" avancé aujourd'hui en sociologie par P.Bourdieu [1994]11. Notons que L.Bazzoli [2000, p.26] revendique pour $\mathrm{J}$.R.Commons un institutionnalisme méthodologique, entre individualisme méthodologique et holisme.

\footnotetext{
10J'ai proposé (Palloix [1997, p.68]) un rapprochement de l'analyse des transactions de J.R.Commons avec les éléments du dispositif de l'économie non standard. L'opération est délicate et suppose un possible rabattement de l'économie politique de J.R.Commons sur l'économie politique non standard.

${ }^{11} \mathrm{Cf}$. par exemple : " "Une des fonctions de la notion d'habitus est de rendre compte de l'unité de style qui unit les pratiques et les biens d'un agent singulier ou d'une classe d'agents ... L'habitus est ce principe générateur et unificateur qui retraduit les caractéristiques intrinsèques et relationnelles d'une position en un style de vie unitaire, c'est-à-dire un ensemble unitaire de choix de personnes, de biens, de pratiques. Comme les positions dont ils sont le produit, les habitus sont différenciés ; mais aussi ils sont différenciants. "(Bourdieu 1994, p.23)
} 
2) L'analyse dynamique et historique de l'institution de T.Veblen (un aller-retour permanent entre les habitudes/règles et les institutions), si elle est le point de départ de celle de J.R.Commons s'enrichit avec ce dernier

- et d'une double dimension nouvelle explicite, celle de la coordination interne et externe des institutions,

- et de la nécessité d'une question liée, celle de l'organisation.

3) Sur le fond, on retrouve le vieux thème de la question de la formation de la totalité à partir de la séparation des individus, question qui hante l'économie politique. Comment le tout tient ensemble ${ }^{12}$ ? L'institution est la réponse de l'O.I. à cette question fondamentale, là où d'autres auteurs dans l'économie politique répondent : " monnaie ".

4) Mais, en retour, "l'institution" se substitue à toute autre analyse pour représenter le "lien social " premier, soit une forte réduction "institutionnaliste " sur l'institution - qui emplit tout - quant à la complexité des rapports entre "société " et "économie ".

\subsection{La radicalité de $T$.Veblen dans la conduite du dispositif sur les questions de l'organisation (C2) et des conflits distributifs (C3)}

2.2.1. Théorie des actifs de T. Veblen: vers une théorie de l'organisation (C2)

A l'arrangement institutionnel se juxtapose une théorie des actifs, qui représente une extériorité, le fonds commun originel de la société comme force collective et qui fait l'objet d'une appropriation privée. La théorie des actifs désigne la question de l'organisation (sous la forme d'une extériorité), présentée comme la solution à une contrainte technologique.

T.Veblen développe une théorie originale des actifs à partir d'un fonds commun originel, posé comme extériorité et enjeu de l'arrangement institutionnel : "Cette connaissance et cette compétence des techniques de vie appartiennent à l'ensemble du groupe; et mis à part les emprunts faits à d'autres groupes, c'est le produit du groupe lui-même, bien qu'il ne soit pas du à une seule génération. On peut les désigner comme l'équipement immatériel, ou,

${ }^{12} \mathrm{Cf}$. l'importance de cette question dans Benetti et Cartelier [1980], Palloix [1981] 
par liberté de langage, comme les actifs intangibles de la communauté. ..... Ce fonds commun de connaissances et de pratiques est peut-être détenu de manière imprécise et sans forme définie; mais, c'est, pourrait-on dire, le groupe dans son ensemble en tant que personnalité collective qui le détient, dans toute son étendue, comme un fonds commun. C'est aussi le groupe qui le transmet et l'accroît, ..." (1908, pp.108-109)

Il en découle un point de vue très restrictif quant à tout apport individuel sur le processus de développement technologique, de création, d'innovation, qui est conçu nécessairement comme un processus plus collectif qu'individuel :

"Les inventions et les découvertes ainsi faites incarnent toujours une telle part de ce qui est déjà donné que la contribution créatrice de l'inventeur est négligeable en comparaison. "(1908, p.111).

La question de l'entrepreneur schumpéterien ne se pose pas : l'entrepreneur du monde des affaires, appelé aussi capitaine d'industrie, est un prédateur.

Au fur et à mesure du développement des actifs matériels (qui viennent et font partie du fonds commun), la question des droits de propriété émerge pour résoudre le principe de régularité temporelle de l'accumulation :

"Mais à mesure que le volume, la portée et l'efficacité du fonds commun de connaissances techniques augmentent, l'équipement matériel qui permet de les mettre en ouvre se développe et devient plus important par rapport aux capacités de l'individu. Et dès que, ou dans la mesure où le développement technique est tel qu'il exige une unité relativement grande d'équipement matériel pour la poursuite effective de l'industrie.... alors un principe de force intervient, les droits de propriété commencent, apparemment, à prendre une forme définie ;... les principes de propriété acquièrent force et consistance et les gens commencent à accumuler les biens capitaux." (1908, pp.113114)

Cela conduit T. Veblen à une mise en cause plus ou moins explicitée du droit de propriété :

"A parler ainsi de la propriété comme d'une institution pour accaparer l'acquis intangible de la communauté, on exprime inévitablement, bien que sans le vouloir, une note de condamnation." (1908, p.117) 
A partir du fonds commun, deux grandes formes d'actifs se déploient sur la base du droit de propriété, actifs tangibles 13 et actifs intangibles.

La question implicite de l'organisation surgit dans la mise en place des actifs et dans leur efficience, comme solution à la contrainte technologique :

"On ne peut en effet poursuivre l'activité industrielle qu'en ayant recours à l'échelle et aux méthodes requises par la technologie, et ceci nécessite un équipement matériel de dimensions données (étendues)" (1908, p.124)

Il est possible d'interpréter comme suit, dans le dispositif marxiste critique de l'économie non standard, les rapports entre fonds commun et théorie des actifs de T. Veblen. Le fonds commun immatériel A est transmis et réifié dans le système d'actifs en capital $\mathrm{A}^{\mathrm{K}}$, selon le schéma ci-après (en régime de valeurs v) avec une valeur actuelle du capital $\mathrm{A}_{\mathrm{v}}{ }_{\mathrm{t}}{ }^{\mathrm{t}}$ qui dérive en dernier ressort de la transmission de valeurs nouvelles par l'actif travail $\mathrm{A}^{\mathrm{T}}$, c'est-à-dire qui dérive de la transmission du fonds commun originel et de son accroissement incessant, alors que $\mathrm{A}^{\mathrm{Kt}-1,} \mathrm{~A}^{\mathrm{Kt}-2}, \mathrm{~A}^{\mathrm{Kt}-\mathrm{n}}$, sont les vecteurs de la transmission du travail mort (matériel, sous forme de connaissances, et réifié) et de son fonds premier. L'extériorité (fonds commun) est dans $\mathrm{A}_{\mathrm{v}}^{\mathrm{k}}{ }^{\mathrm{t}-\mathrm{n}}$ tout comme dans $\mathrm{A}^{\mathrm{T}}$.

Les encadrés désignent dans la figure 4 ci-après l'extériorité du fonds commun tout comme la prétention erronée de l'économie politique à l'endogénéisation de toutes ses composantes.

Figure 4 : Formation des actifs et fonds commun

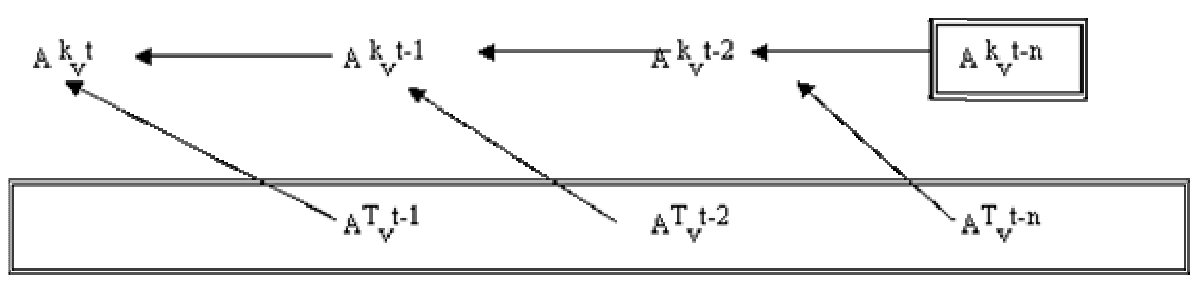

Pas d'actif, si les institutions ne sont pas capables de mobiliser une puissance - le fonds commun de la société -, si les institutions

13 "Les actifs tangibles doivent leur capacité de production et leur valeur aux moyens industriels immatériels qu'ils incarnent ou que des propriétaires peuvent accaparer en vertu de leur propriété. Ces moyens industriels immatériels sont nécessairement un produit de la communauté, le résidu immatériel de son expérience, passée et présente, expérience qu'on ne peut séparer de la vie de la communauté et qui ne peut être transmis que sous sa garde. " $(1908$, p.128) 
ne confèrent pas une forme organisationnelle à cette capture. La question de l'organisation surgit implicitement dans la capture des actifs et leur mise en œuvre productive.

\subsubsection{Les conflits distributifs chez T.Veblen (C3)}

En économie politique, l'émergence de tout conflit distributif s'enracine bien évidemment dans une théorie des prix. G.Hodgson [1998, p.169] soutient que la théorie des prix, chez T.Veblen, s'apparenterait davantage à une théorie marshallienne qu'à une théorie ricardienne ou smithienne. La vivacité des conflits distributifs chez cet auteur suppose de fait un recours implicite à une théorie classique du prix comme modalité de répartition.

Un premier conflit distributif est lié à la structure de classe de la société, avec une distribution inégalitaire des richesses :

"L'accumulation des richesses à l'extrémité supérieure de l'échelle sociale implique la privation à l'extrémité inférieure. "(1899, p.134)

Le deuxième conflit distributif, le plus important pour T.Veblen, est celui qui oppose le monde des affaires - l'économie marchande et financière dans ma conceptualisation [Palloix 1996] à celui de l'industrie, avec transfert de richesses de l'industrie vers le monde des affaires.

Après avoir rappelé la corrélation classique entre les trois facteurs de production - terre, travail, capital - et les trois catégories de la répartition - rente, salaire, profit -, soit une vision très ricardienne du système de prix, T.Veblen constate l'absence d'un facteur de production tout aussi important, les " arts industriels "14, car non corrélé à un revenu, et qui est le principal opérateur de la création de la richesse sociale :

"Cet état des arts industriels est un fonds commun de savoir hérité de l'expérience passée, détenu et transmis comme une propriété indivise de la communauté entière. Il constitue la base indispensable de toute activité productive, et, à part quelques infimes fragments protégés par des brevets ou des secrets de fabrication, ce fonds commun n'est la propriété individuelle de personne. Aussi ne l'a-t-on pas compté comme facteur de production. Les progrès technologiques sans précédent, réalisés au cours des cent cinquante dernières années, ont commencé à attirer l'attention sur cet oubli dans le

${ }^{14}$ C'est la grande illusion de T.Veblen. Les logiciels, bases de données, internet, ... autorisent aujourd'hui une appropriation privée croissante du fonds commun. 
schéma tripartite des facteurs de production, hérité de l'époque précédente." (1921, pp.17-18)

La création de la richesse sociale par les "arts industriels " n'est pas conservée par l'industrie au bénéfice possible du plus grand nombre, mais est transférée à la classe de loisir, au monde des affaires.

L'actualité de l'opposition entre le monde des affaires et celui de l'industrie reçoit un éclairage prémonitoire dans le mode de gestion "financier " de l'industrie ${ }^{15}$. Pour T.Veblen, le seul regard du monde des affaires sur le monde industriel est celui des prix, et non de la valeur et de la valeur d'usage dira-t-on. La logique du monde des affaires est construite sur celle d'une stricte évaluation par les prix au profit de la rémunération financière, sans autre égard à la logique productive industrielle :

"A force de se consacrer à une évaluation stricte et rigoureuse de toute chose en terme de prix et de profit, ils deviennent incapables d'apprécier les faits et les valeurs technologiques qui ne peuvent être formulés qu'en terme de résultats mécaniques concrets ....

Ce sont des spécialistes des prix, des profits et des opérations financières; et pourtant ils ont toujours le dernier mot sur tous les problèmes de politique industrielle. Ce sont des capitaines financiers, par formation et par intérêt, et cependant, bien qu'ils n'aient aucune compétence dans les arts industriels, ils continuent d'exercer un pouvoir discrétionnaire entier comme capitaines d'industrie. "(1921, p.34)

L'hégémonie du monde des affaires ${ }^{16}$ sur l'industrie conduit à un gaspillage des ressources, à une sous-utilisation des potentialités de l'industrie.

L'utopie de T.Veblen quant au monde des ingénieurs ${ }^{17}$, aux potentialités d'organisation qu'ils représentent, le conduira à en appeler à " un soviet des ingénieurs".

${ }^{15}$ Qui s'exprime par exemple dans les modalités du "contrôle de gestion " en entreprise.

16 "... les affaires se sont développées à un tel point, que l'on peut légitimement se demander si dans leur gestion les capitaines ne s'occupent pas plus du freinage de l'industrie que de l'accroissement de sa productivité. " (1921, p.19)

17 "... la dictature industrielle du capitaine financier ne se maintient que par la tolérance des ingénieurs et elle est passible à tout moment d'être interrompue, à leur gré et comme il leur conviendra. " (1921, p.50) 


\section{3. "Le capitalisme raisonnable" de J.R.Commons (C2/C3)}

\subsubsection{Action collective, institution, organisation (C2)}

Les concepts d'échange et d'actif s'effacent devant celui de "transaction" qui les rassemble, les multiplie, les enrichit dans le dispositif de J.R.Commons. Les transactions, comme l'indique L.Gillard [2001], s'interprétent comme des transferts de droits de propriété modifiant "les positions créditrices et débitrices des uns par rapport aux autres" (p.142).

La validation de l'institution s'exprime dans la capacité du "going concern" ou action collective, qui est donnée comme partie intrinsèque de celle-ci :

"Nous pouvons définir une institution comme une action collective tournée vers le contrôle, la libération et le développement de l'action individuelle. Les actions collectives s'inscrivent sous toutes les formes depuis la coutume inorganisée jusqu'à la plupart des affaires organisées, telles que la famille, l'entreprise, le syndicat, la banque centrale, l'Etat. Le principe commun à toutes ces formes est le plus ou moins grand degré de contrôle, de libération et de développement de l'action individuelle par l'action collective" (1932, p.649).

L'action est organisation, l'institution mettant en oeuvre une organisation (action) collective, libérant la capacité d'organisation (action) individuelle de l'individu, le tout s'enrichissant des potentialités individuelles et réciproquement.

Tout parait tenir ensemble de façon indissolublement liée : règles avec institutions, règles avec action collective, action collective avec institution, action collective avec organisation, et institution avec organisation.

L'action collective s'exerce à travers des règles qui définissent les relations liées et réciproques entre les individus :

"Tant l'Etat que l'entreprise, ou le cartel, ou la holding, ou la coopérative, ou le syndicat....se doit de poser et appliquer les règles qui déterminent pour les individus un ensemble de relations économiques liées et réciproques. " (1932, p.649)

Mais, en même temps, les actes collectifs imposent en retour leurs règles: "tous les actes collectifs établissent des relations de droits et de responsabilités, de non droits et de non responsabilités. " (1932, p.649) 
L'action collective apparaît comme le guide et la libération des potentialités de l'individu :

"Cette action collective est plus que le contrôle de l'action individuelle. C'est, à travers l'acte véritable de contrôle, une libération de l'action individuelle de toute forme de coercition, de contrainte, de discrimination ou de concurrence déloyale conduite par d'autres individus.

Et l'action collective est plus que le contrôle et la libération de l'action individuelle : c'est le développement d'un projet volontaire de l'individu plus loin que nous ne pourrions le conduire à l'aune de notre seule capacité" (1932, p.651).

L'institution est action collective pour et au service du contrôle, de la libération et de l'expansion de l'action des individus.

Règles (Institutions) + Action collective $=$ Organisation, $\mathrm{du}$ moins telle semble être l'équation de J.R.Commons: "Ces actes collectifs des organisations économiques ..." (1932, p.649), tout en rappelant qu'il est difficile de dissocier ces éléments.

Face à cette liaison apparemment organique, si on l'accepte, force est de se rallier à l'élégante formulation de L.Bazzoli [2000] :

"L'analyse que propose Commons de l'action collective organisée et de ses caractères spécifiques à travers le concept de going concern a ceci de particulier qu'elle vise à capturer à la fois la face organisationnelle des institutions et la face institutionnelle des organisations. "(p.105)

Le doute subsiste face à une construction où tout est dans tout : l'action collective de J.R.Commons apparait comme un deus ex machina, qui n'est pas sans analogie avec la mobilisation du fonds commun de T.Veblen, sous une forme organisationnelle, par l'institution. L'action collective ${ }^{18}$ de J.R.Commons, tout comme l'organisation de T.Veblen, paraît demeurer une extériorité en dépit d'un collage volontariste avec l'institution.

\footnotetext{
${ }^{18}$ Indépendamment des rapports avec T. Veblen (processus vital, fonds commun, théorie des actifs), la conceptualisation de J.R.Commons offre quelque similitude avec le travailleur collectif de K.Marx (Cf. "Le Chapitre inédit du Capital "), où le tout est supérieur à la somme des parties.
} 
Les conflits ne sont pas donnés comme des conflits de répartition, mais éventuellement comme des conflits liés aux coutumes (inorganisées), et que les institutions ont vocation à résoudre.

Plus qu'un conflit distributif, l'économie est donnée comme duale, comme une relation particulière et une tension entre la création de "valeurs d'usage " d'un côté, et la création de "valeurs de rareté " ou valeurs d'échange de l'autre [Bazzoli 2000, p. 151]. La théorie de la valeur et des prix ne sont pas des variables de répartition, mais des variables appartenant au champ institutionnel et organisationnel (going plant, going business).

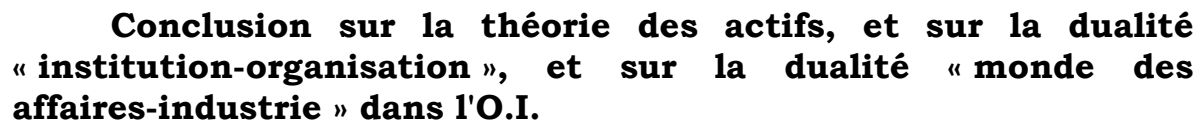

1) Les institutions ne portent pas seulement sur la coordination des agents, mais aussi sur les actifs génériques (le fonds commun) et leur appropriation sous forme d'actifs tangibles et intangibles. Là où l'institution autorise l'appropriation des actifs (en provenance $\mathrm{du}$ fonds commun) en tant qu'actifs spécifiques (y compris dédiés) et leurs cessions, elle se double nécessairement d'une hypothèse de nomenclature des actifs pour leur valorisation sous forme " prix".

2) L'arrangement institutionnel apparait comme la forme (le cadre) de la capture de la puissance productive des actifs (capital et travail), ne serait-ce qu'au vu des droits de propriété, du contrat de travail, ce qui suppose que surgisse l'organisation - un arrangement organisationnel - comme modalité d'exercice de la puissance des actifs dans la création des richesses.

3) L'organisation désigne une extériorité de l'économie politique dans l'institutionnalisme historique, la puissance de la société mobilisée par les institutions et l'appropriation des actifs. L'organisation des actifs en tant que puissance productive susceptible d'être captée par l'arrangement institutionnel - suppose, elle aussi, sa propre hypothèse de nomenclature, une théorie de la 
valeur ${ }^{19}$ (comme puissance du fonds commun) et de son utilité sociale (la valeur d'usage). L'O.I., dans la conduite de son dispositif de recherche, dévoile également l'inanité de toute tentative de passage de la valeur (l'organisation) au prix (l'arrangement institutionnel).

4) Les théories de T.Veblen et J.R.Commons se présentent, dans leurs différences, comme des tentatives de construction d'une théorie générale, articulée à une théorie des prix et de la monnaie. Il aurait d'ailleurs été fort judicieux de prolonger l'analyse du dispositif global de l'institutionnalisme vers une combinaison $\mathrm{C} 4$ sur la question de la "monnaie ", quant à sa prise en compte ou non, quant à son endogénéisation ou son extériorité. Mais ceci dépasserait notre propos.

5) Il est assez remarquable de relever que l'opposition du monde des affaires (à l'activité de prédateur) et de l'industrie, que pointe T.Veblen dès 1899, parcourt l'économie politique dans la transition du XIXème au XXème siècle, et se retrouve chez le ricardien J.A.Hobson [1902], le marxiste R.Hilferding20 [1910], et curieusement chez l'industriel H.Ford ${ }^{21}$ [1926]. Cette opposition, si décriée et ridiculisée un moment, apparaît particulièrement judicieuse dans la phase actuelle du capitalisme, où le maître mot de la gestion moderne des entreprises est "la création de valeurs pour l'actionnaire ", pour le marchand financier [Palloix 1996].

\section{Un dispositif institutionnaliste plus éclectique dans la N.E.I. : Institutions et organisations}

Si M.Rutherford [1994] soutient que la N.E.I. se déploie sur deux grands courants, une économie standard marginaliste orthodoxe (de filiation walraso-parétienne) d'un côté, et une économie standard plus hétérodoxe (d'inspiration autrichienne) :

"Il est possible de distinguer un courant davantage néoclassique et un courant davantage autrichien dans la NEI. La majorité des travaux de la NEI appartiennent au premier courant. Le second courant comprend les travaux de Langlois, avec une configuration ancrée dans les travaux de Hayek sur les institutions, les travaux de

${ }^{19} \mathrm{Ce}$ qui en appelle à une théorie élargie de la valeur "travail " vers la valeur "connaissances", dans un processus de déclaration - et non d'autodéclaration comme dans l'économie politique - de la société sur ce fonds commun.

${ }^{20}$ Cf. Palloix (1999)

${ }^{21}$ C'est ainsi qu'on peut relever ce propos d'H.Ford sur le monde financier : "L'argent investi sans risque, et qui prélève sa rançon n'est pas de l'argent actif " $(1926$, p.39) 
Nelson et Winter dans une perspective évolutionniste néoschumpéterienne, et probablement certains travaux en théorie des jeux." (p.3),

ceci n'est pas le point de vue de G.Hodgson par exemple, d'autant plus qu'il ne rattache pas l'école autrichienne à l'économie standard.

Il est également plus ou moins admis le caractère tout aussi hétéroclite de la N.I.E. que celui de l'O.I. : "Il est probablement vrai que la NEI est tout aussi disparate que l'O.I... " (Rutherford 1994, p.2), avec des couches successives apportées, entre autres, par la théorie des droits de propriété (Demsetz [1967], Alchian et Demsetz [1973]), la théorie de l'agence (Jensen et Meckling [1976]), la théorie des coûts de transaction de Coase [1937], la théorie des contrats d'O.E. Williamson [1975,1985,1996], la théorie des jeux (Schotter [1981]), débouchant sur une histoire économique institutionnaliste [North 1981,1990], dans un contexte de relâchement des hypothèses du dispositif de 1'E.E.G.

L'éclectisme du courant institutionnaliste, dans une version "détour et retour " vers l'économie standard, tient certainement au problème de sa cohérence avec une théorie des prix et de la monnaie, qui est empruntée telle quelle (par exemple, à la théorie marshallienne du prix, à celle du mark-up, à celle du prix walrasien), faute d'une genèse endogène de sa propre théorie.

3.1. Un dispositif relativement commun de la N.E.I. sur l'arrangement institutionnel (C1), mais davantage rabattu sur l'individualisme méthodologique des comportements

Le comportement de l'agent dans la NEI, élément central de l'analyse économique, s'écarte peu des fondements de l'économie standard. L'agent est un individu rationnel, même si, en information limitée et imparfaite, une rationalité procédurale se substitue à la rationalité substantive de l'économie standard. Les institutions apportent les régularités de comportements, au vu d'une boucle action-information (cf. Figure 1) :

"La caractéristique du nouveau projet institutionnaliste est la tentative d'expliquer l'émergence des institutions, telle que la firme ou l'Etat, en relation à un modèle de rationalité ...Le mouvement explicatif court des individus aux institutions, prenant les individus comme donnés. Cette approche est souvent désignée sous le vocable d'individualisme méthodologique....

Nous pouvons en donner une représentation sous la forme d'une boucle action-information." (Hodgson [1998], p.176). 
3.2. Arrangement institutionnel dans la N.E.I. et l'impossible question de l'organisation (C2)

La matrice institutionnelle de D.C.North apparait comme l'exposé le plus élégant de l'arrangement institutionnel dans la N.E.I. Une matrice institutionnelle (D.C. NORTH [1990]) est un ensemble de règles formelles et de contraintes informelles qui structurent les interactions entre les individus ${ }^{22}$. Les règles formelles ou les contraintes informelles sont réunies sous le vocable d'institutions. Par ailleurs, la matrice institutionnelle permettrait de distinguer, tout en les reliant, les règles du jeu (institutions) et les joueurs (organisations). Cette distinction, très séduisante à première vue, ne l'est guère sur le fond car elle apparaît comme une déclinaison de la figure 1, les agents devenant des "joueurs" ( qui font face aux institutions : les règles formelles et informelles) .... dans l'organisation, mais celle-ci venant d'où ?

La faille du dispositif northien tient au non dit sur les enjeux de l'arrangement institutionnel: l'arrangement institutionnel ou matrice institutionnelle est réduit à deux composantes, les comportements (agents, joueurs) et les institutions ou règles; la composante "actifs" de l'I.H. a disparu, privant la matrice de tout enjeu portant sur les actifs.

L'arrangement institutionnel d'O.E.Williamson [1985, 1996] est plus complexe, plus éclectique, car les emprunts sont nombreux dans une tentative de synthèse et d'approfondissement de tous les courants de la N.E.I. O.E.Williamson apparaît comme un grand " assembleur". L'arrangement institutionnel se déploie sur institution, transaction, contrat, droit de propriété, actif ${ }^{23}$, organisation et structure de gouvernance, avec une multiplicité de modes de coordination ${ }^{24}$ (du prix à la firme, au réseau, à la coopération, ...), tout en intégrant la théorie de l'agence. La question organisationnelle ne se pose pas : l'organisation est un emprunt aux théories de l'organisation en gestion des entreprises (notamment à Chandler), en dehors de toute genèse endogène "williamsonnienne " qui lui soit propre. Dès lors le programme de recherche de la " comparative institutional analysis $" 25$ porte pour partie sur la tentative de refonder la cohérence globale du dispositif, notamment

${ }^{22} \mathrm{Cf}$. la présentation de la matrice institutionnelle de D.C.North dans L.Kichou [2001] ${ }^{23}$ Par exemple, l'analyse de la spécificité des actifs (qui ne dépend que de la fréquence) dans la théorie de O.E. Williamson apparaît fort limitée, car cantonnée à la seule redéployabilité (ou non) de l'actif, soit un rabattement de la spécificité sur une analyse purement transactionnelle (cf. Berquez et Girard [2000] et Girard [1999]).

${ }^{24}$ Cf. la présentation des différents types de coordination chez O.Weinstein [1997]

${ }^{25}$ Selon l'expression de R.H.Coase [1964] 
dans une version "économie des contrats "26 [Brousseau 1993], qui se dote d'une analyse de "l'incomplétude ", pour tenter de basculer de l'institution vers l'organisation, mais sans résultat probant jusqu'ici.

Observons que c'est dans le cheminement de l'hétérodoxie en économie politique que naît la question de la distinction entre institution et organisation : comment l'arrangement institutionnel de la valorisation dans l'O.I. capture l'efficience, la puissance de la société détenue dans des actifs (tangibles, intangibles, humains), ce qui - par rapport à des extériorités - nécessite l'organisation. L'orthodoxie, en économie standard et non standard, ne reconnaît aucune extériorité, d'où une difficulté existentielle d'analyse de l'organisation, que n'a pas la discipline de la gestion d'entreprise. La discipline de la gestion d'entreprise, qui n'est prisonnière d'aucune hypothèse de nomenclature (pas de question sur la monnaie et les prix), d'aucune hypothèse sur la formation de la totalité, est libre de s'adonner à la connaissance de l'organisation, comme le fait remarquablement H.Mintzberg (1982) par exemple. Par contre, la question de l'organisation en économie politique standard et non standard est apparemment insoluble dans le champ de l'orthodoxie, à moins ..... de déshabiller l'institution au profit de l'organisation.

C'est cette dernière solution que l'on retrouve chez C.Ménard [1993] qui propose une définition très restrictive de l'institution, dépourvue de toute capacité de coordination (des prix à la coopération,...) : "les institutions, à la différence des marchés ou des organisations, ne sont pas des mécanismes de coordination à proprement parler; elles contribuent à définir les conditions sociohistoriques dans lesquelles ces mécanismes peuvent s'instaurer. "(p.15). Par contrecoup, l'organisation se pare des attributs enlevés à l'institution: "une organisation économique est une procédure de coordination spécifique. "(p.15). L'organisation entre dès lors dans le champ de l'économie politique de la N.E.I., et c'est là le but de l'opération, tout en révélant la grande difficulté de l'exercice.

Il n'en demeure pas moins que la N.E.I. offre des cadres d'analyse (institution, actif, économie contractuelle, modes de coordination, structure de gouvernance, organisation, droits de propriété, ...), peut-être fort éclectiques, mais non dénués d'une certaine capacité à parcourir les terrains de l'économie et de l'entreprise.

\footnotetext{
${ }_{26}$ "L'économie des contrats est une transition vers une entière reconsidération de la spécificité " (Berquez et Girard [2000, p. 10])
} 
Par exemple, la gouvernance d'entreprise peut se déployer sur deux champs distincts et apparemment complémentaires :

- celui de l'arrangement institutionnel qui porte sur les droits de propriété, une économie contractuelle des transactions (et de leur incomplétude ${ }^{27}$ ), la spécificité des actifs et leur valorisation, les modes de coordination propres à l'arrangement institutionnel, sur la base néanmoins incontournable d'une hypothèse de nomenclature affichée en prix ;

- celui de l'arrangement organisationnel qui vise l'efficience de la mise en œuvre productive de la spécificité des actifs ${ }^{28}$, s'appuyant sur la mise en place de dispositifs $\operatorname{cognitifs}^{29}$, avec ses modes de coordination propres (hiérarchique, coopération, réseau, ...), hors de toute hypothèse de nomenclature ${ }^{30}$.

Mais la difficulté théorique tient à l'empilement, à la juxtaposition de deux points de vue sur l'entreprise, qui sont ceux de l'économie politique d'un côté (standard ou non standard, peu importe) et de la gestion d'entreprise, sociologie des organisations, ... de l'autre. A moins de se placer dans le champ de la critique de l'économie politique, mais, c'est un autre programme de recherche.

\subsection{Le dispositif institutionnaliste de R.N.Langlois et la question endogène de 1'organisation (C2)}

R.N.Langlois poursuit deux objectifs très clairement énoncés, pallier d'un côté aux défaillances du modèle d'équilibre économique général walraso-parétien en recourant à l'analyse de Hayek (1990a, p.8), et de l'autre rejeter toute analyse de l'organisation de type S.C.P. (Stratégie-Comportement-Performance) par exemple empruntée à l'analyse de la gestion des entreprises ${ }^{31}$ faute de fondement théorique d'une telle analyse (1990a, p.10).

${ }^{27}$ Cf. F.Aboubeker et L.Kichou [1999]

28 ce qui conduit E.Brousseau [1993] à proposer de passer d'un point de vue allocatif de la spécificité des actifs (arrangement institutionnel) à un point de vue productif (arrangement organisationnel).

${ }^{29} \mathrm{Cf}$. B.J.Loasby [1990]: "Une organisation doit être analysée comme un "établissement éclairé " de scientifiques. ... L'efficience organisationnelle requiert la spécialisation des savoirs et routines, et cette spécialisation entraîne l'apprentissage de signaux particuliers et de réponses - une ossature particulière pour interpréter les événements" (p.51)

${ }^{30}$ Le contrôle de gestion vise à résoudre cette question en ramenant un système de prix avec des clefs de répartition entre les actifs (une hypothèse de nomenclature extérieure) pour éclairer les boîtes noires de l'entreprise.

${ }^{31}$ R.Langlois paraît faire une exception pour Chandler (1990a, p.19), mais peut-être pour une toute autre raison. 
L'apport de R.N.Langlois porte sur la distinction de deux grands types d'institutions, l'institution organique, l'institution pragmatique (d'après Menger), soit un essai d'enracinement dans l'école autrichienne: "une norme sociale est dans un certain sens différente d'une institution sociale telle qu'une entreprise. Dans les termes d'Hayek (1973), les règles de conduite contenues dans la première sont "abstraites", s'appliquant à un large champ de personnes et d'activités, tandis que le corps de règles qui gouverne une entreprise industrielle est tourné vers des buts spécifiques. Mais tous deux (Menger, Hayek) les qualifient d'institutions. " (p.19).

Les fondements de l'analyse, comme le reconnaît très clairement R.N.Langlois, sont apportés :

- d'un côté, par la théorie de la monnaie de C.Menger "monnaie par laquelle, les actions intéressées d'échange réciproques conduisent d'une économie de troc à une économie complexe où un simple bien est devenu le moyen d'échange universel." (1990b, p.248), tout en sachant que ce n'est pas la question de la monnaie qui intéresse Langlois, mais les conditions de son émergence,

- de l'autre, par l'emprunt à la théorie hayékienne de l'autoorganisation : "L'autre approche ... s'enracine, non dans le processus par lequel la structure émerge, mais dans le processus qui maintient la structure une fois celle-ci établie." (1990b, p.248)

Dès lors, arrangement institutionnel et arrangement organisationnel peuvent se concevoir comme deux modes organiquement liés; si le point de départ est un état institutionnel pragmatique dans le contexte général de l'économie contractuelle, son évolution institutionnelle est un processus organisationnel (R.N.Langlois [1990a, p.19]). L'institution organique est l'expression d'un processus d'auto-organisation qui s'enracine d'emblée dans l'institution pragmatique.

La cohérence du dispositif proposé par R.N.Langlois paraît dépendre d'une institution première convoquée, la monnaie, comme expression d'un processus d'interaction et d'auto-organisation, même si ce n'est pas là le but de l'auteur. 


\section{Conclusion}

\section{1) Quel(s) passage(s) entre "institution " et " organisation"?}

Institution et organisation campent dans deux champs théoriques différents.

L'institution, comme composante de l'arrangement institutionnel, s'inscrit dans les différents courants (l'O.I. de T.Veblen et de J.R.Commons, la NEI de la "comparative institutional analysis ", l'école autrichienne) d'une économie politique (non standard et standard) qui lui donnent, par définition, des éclairages différents, avec des emprunts réciproques (droits de propriété, spécificité des actifs, transactions, contrats), mais aussi des oppositions, le conflit majeur reposant sur l'analyse des comportements (holisme-individualisme, institutionnalisme méthodologique, individualisme méthodologique ${ }^{32}$ ). Cet arrangement institutionnel s'inscrit, au-delà des divergences sur les processus de formation de la valeur et des prix, dans un objectif essentiel, celui de la valorisation.

Certes, les différents courants de l'institutionnalisme donnent tous un même objectif à l'arrangement organisationnel, celui de l'efficience de la spécificité des actifs, avec des éléments utilisés en commun (hiérarchie, spécificité des actifs, ...). Mais, sous ce dénominateur commun, malgré la prétention de certains courants (l'O.I. de J.R.Commons, la NEI de R.N.Langlois), l'arrangement organisationnel échappe à l'arrangement institutionnel pour se déployer dans un champ autre que celui de l'économie politique, hors de l'hypothèse de nomenclature. Le rabattement de l'organisation dans le champ de l'arrangement institutionnel n'est que solution de facilité et n'emporte pas preuve de la validité du dispositif de la N.E.I. de la "comparative institutional analysis" comme cela apparait par exemple chez R.H .Coase ${ }^{33}$. L'arrangement organisationnel véhicule une extériorité, un emprunt (le fonds commun, les connaissances et savoirs, la force productive matérielle et immatérielle selon les écoles de pensée) à la société pour la mise en ouvre de l'arrangement institutionnel.

\footnotetext{
${ }^{32}$ avec les relâchements sur la rationalité (de la rationalité substantive à la rationalité procédurale) et sur l'information (d'une information parfaite à une information limitée) ${ }^{33} \mathrm{On}$ trouve très nettement dans les propos ci-après la confusion organisationnelle exprimée sous forme institutionnelle d'allocation des activités : "Selon moi, ce dont on a besoin pour traiter de l'organisation industrielle, c'est une approche directe du problème.....Quelles sont les activités que l'on tend à regrouper, et celles qui ne le sont pas? " (R.H.Coase, p.88)
} 
Le but ultime de l'arrangement institutionnel est cette capture d'une force, mise en œuvre comme organisation, au sein de l'institution.

Bien sûr, on ne passe pas entre "institution " et " organisation", parce que cela relève - peut-être - du même problème que celui du passage (impossible) de la valeur (travail) aux prix de production et aux prix de marché dans l'économie non standard.

\section{2) Que faire de l'organisation?}

Compte tenu de la dualité (et du non passage) de l'arrangement organisationnel et de l'arrangement institutionnel, la tentation est grande

- soit de déshabiller l'institution au profit de l'organisation (Ménard [1993]),

- soit de se déployer uniquement au sein de l'arrangement organisationnel, en recherchant les fondements d'une théorie propre et spécifique de l'organisation ${ }^{34}$, l'organisation apparaissant comme une forme autre.

Il est clair qu'on ne peut se passer d'une approche organisationnelle en stratégie des entreprises. Ce qui a de l'importance dans les groupes multinationaux, c'est l'organisation productive, commerciale, financière qui prend le pas sur l'institution. L'organisation assure l'efficience des actifs (dans les institutions). Curieusement, c'est l'organisation qui est multinationale, globale, et non l'institution.

En contrepartie, les institutions autorisent la répartition des fruits de l'organisation (productive, commerciale, financière), la légitiment (économie de prédation en rentes, profits). Du point de vue des droits de propriété, seule l'institution importe. En effet, seul l'espace des institutions ouvre sur une théorie des prix, ce que ne saurait faire une théorie de l'organisation qui ouvre au mieux sur une théorie de la valeur en économie non standard de type marxiste. L'espace des institutions est davantage national, tout en notant que s'ouvre un espace international de celles-ci.

${ }^{34}$ C'est le but poursuivi, me semble-t-il, dans les travaux de Yorgos Rizopoulos [1999]. 


\section{Bibliographie}

ABOUBEKER Fethi, KICHOU Lyazid (1999), "Transition economies and incompleteness of contracts", Communication au colloque European Integration and Economies in Transition, Department of Business Administration, University of the Aegean (Chios - Greece), December.

BAZZOLI Laure (1994), Action collective, travail, dynamique du capitalisme : fondements et actualité de l'économie institutionaliste de J.R. Commons, Thèse de doctorat, Université Lumière Lyon 2 E.C.T., 430 pages

BAZZOLI Laure (2000), L'économie politique de J.R. Commons, Paris, L'Harmattan, 234 pages

BAZZOLI L., DUTRAIVE V. (1998), Une conception institutionnaliste de l'organisation comme institution - Eléments sur l'apport de J.R. Commons, Communication aux Journées d'Etudes des 12-13 mai 1998 "Institutions et Organisations ", E.R.S.I. - U.P.J.V.

BENETTI Carlo, CARTELIER Jean, (1980), Marchands, salariat et capitalistes, Paris, François Maspéro - Intervention en Economie Politique, 207 pages

BERQUEZ Angélique, GIRARD Jean-Louis (2000), De la théorie des actifs à l'économie factorielle: pour une lecture sensuelle de l'espace, C.R.I.I.S.E.A., U.P.J.V., Communication au séminaire du C.R.I.I.S.E.A., à paraître

BOURDIEU Pierre (1994), Raisons pratiques - Sur la théorie de l'action, Paris, Seuil, 244 pages

BROUSSEAU Eric (1993), L'économie des contrats - Technologies de l'information et coordination interentreprises, Paris, P.U.F., Economie en liberté, 368 pages

CHAVANCE B. (1998), Genèse, évolution et transformation des règles : la hiérarchie constitution/institution/organisation, Communication aux Journées d'Etudes des 12-13 mai 1998 "Institutions et Organisations ", E.R.S.I. - U.P.J.V.

COASE Ronald H. $(1937,1946,1960)$, La firme, le marché et le droit, Paris/New-York, Diderot Editeur, Arts et Sciences, 1997, 276 pages

COMMONS John R. (1899-1900), A sociological view of sovereignty, The American Journal of sociology, Vol. 5 (july-november 1899), pp. 1-15, pp. 155-71, 347-66 (january-may 1900), pp. 544-52, 683-95, 814-25 ; Vol. 6 (july 1900), pp. 67-89 
COMMONS John R. (1932), Institutional economics, American Economic Review (A.E.R.), Vol. 21, december, n4 p. pp. 643-657

COMMONS John R. (1934), Institutional Economics, Its Place in Political Economy, New-York : The Mac Millan Company. Réédition en 1990 : New Brunswick, Transaction Publishers, Vol. 1 \& 2, 921 pages

COMMONS John R., (1935), Le problème de la corrélation du droit, de l'économie et de la morale, dans Les sources $d u$ droit - En l'honneur de François Geny, Paris, Recueil Sirey, Tome 2, pp. 124144

CORIAT Benjamin, WEINSTEIN Olivier (1995), Les nouvelles théories de l'entreprise, Paris, Librairie Générale Française - Le Livre de poche, 218 pages

FORD Henry (1926), Propos d'hier pour aujourd'hui, Paris, Masson, 1992, 212 pages

GILLARD Lucien (2001), Le modèle Commons d'économie transactionnelle, Cahiers d'Economie Politique - Histoire de la Pensée et Théories, $\mathrm{N}^{\circ} \mathrm{s}$ 40-41 : Lectures de John R.Commons, L'Harmattan, pp. $139-175$

GIRARD Jean-Louis (1999), De la théorie des actifs à l'économie factorielle: travail et économie marchande dans l'économie urbaine, E.R.S.I., Université de Picardie Jules Verne, Thèse de doctorat, 562 pages

HILFERDING Rudolf (1910), Le capital financier - Etude sur le développement récent du capitalisme, Paris, Les Editions de Minuit, 1970, 478 pages

HODGSON Geoffrey M. (1998), The approach of institutional Economics, Journal of Economic Literature, Vol. 36, March 1998, pp 166-192

HODGSON Geoffrey M. (2000), Evolution and Institutions - On evolutionnary Economics and the Evolution of Economics, Edward Elgar Publishing Ltd, Cheltenham U.K.,345 pages

KICHOU Lyazid, PALLOIX Christian (1999), La firme algérienne sous double gouvernance: nationale et mondiale, Communication au Colloque international de Ghardaïa, 25-27 janvier 2000, à paraître

KICHOU Lyazid, Institutions et organisations : réformes économiques et processus de privatisation en Algérie - 1990-2001, Thèse de doctorat, UPJV-CRIISEA Amiens, 2001, 320 pages

KLEIN Peter G. (1998), New Institutional Economics, Athens, University of Georgia, doc. roneo, july, 47 pages 
LANGLOIS Richard N. (1990a), The new institutional economics : an introductory essay, pp. 1-25, dans Richard N.Langlois Ed., Economics as a process - Essays in the new institutional economics, Cambridge University Press, 1990

LANGLOIS Richard N. (1990b), Rationality, institutions, and explanation, pp. 225-255, dans Richard N.Langlois Ed., Economics as a process - Essays in the new institutional economics, Cambridge University Press, 1990

LOASBY Brian J. (1990), Organisation, competition, and the growth of knowledge, pp. 41-57, dans Richard N.Langlois Ed., Economics as a process - Essays in the new institutional economics, Cambridge University Press, 1990

LONGUET Stéphane (1998a), Hayek et l'école autrichienne, Paris, Nathan, 192 pages

LONGUET Stéphane (1998b), Lachman et Hayek: deux conceptions de la dimension institutionnelle chez les autrichiens, Communication aux Journées d'Etudes des 12-13 mai 1998 "Institutions et Organisations ", E.R.S.I. - U.P.J.V.

MARX Karl (1862-63), Un chapitre inédit du capital, Paris, Union Générale d'Editions 10/18, 1971, 319 pages

MENARD Claude (1993), L'économie des organisations, Paris, Repères, La Découverte, 127 pages

MINTZBERG Henry (1982), Structure et dynamisme des organisations, Paris, Les éditions d'organisation, 434 pages

NORTH D. C. (1990), Institutions, Institutional Change, and Economic Performance. New York, Cambridge University Press.

NORTH D. C. (1994), Economic Performance Through Time, American Economic Review, vol. 84, pp. 359-368, June.

PALlOIX Christian (1981), De la socialisation, Paris, François Maspéro - Intervention en Economie Politique, 192 pages

PALLOIX Christian (1996), Société et Economie - L'industrie et les marchands, Paris, L'Harmattan, 240 pages

PALLOIX Christian (1997), Eléments pour une théorie hétérodoxe de l'économie industrielle, dans C.PALLOIX \& Y.RIZOPOULOS (1997), pp.55-74

PALLOIX Christian, RIZOPOULOS Yorgos Coord. (1997), Firmes et Economie Industrielle, Paris, L'Harmattan, 446 pages

PALLOIX Christian (1999), Monnaie de crédit et capital financier chez Rudolf Hilferding, CRIISEA, U.P.J.V., Communication aux Journées 
d'Etudes 19-21 mai 1999 sur "Les Economistes Autrichiens 19711939 ", à paraître

RIZOPOULOS Yorgos (1999), Stratégies organisationnelles et réseaux post-socialistes en Russie, Revue d'Etudes Comparatives Est-Ouest, Vol. 30, n 2-3, pp. 283-302

RUTHERFORD Malcolm (1994), Institutions in Economics - The old and the new Institutionalism, Cambridge University Press, 225 pages, 1994

SAMUELS Warren J. (1995), The present state of institutional economics, Cambridge Journal of Economics, n 19 , pp.659-590

SRAFFA Piero (1960), Production de marchandises par des marchandises - Prélude à une critique de l'économie politique, Paris, Dunod, 1970, 124 pages

VEBLEN Thorstein (1899), Théorie de la classe de loisir, Paris, Gallimard - TEL, 1978, 278 pages, précédé de Avez-vous lu Veblen ? par Raymond Aron

VEBLEN Thorstein (1908), Nature du capital (Q.J.E., Harvard University Press), dans Thorstein Veblen, Les ingénieurs et la capitalisme, Paris, Gordon \& Breach - Publications Gramma, , 1971, pp.105-162

VEBLEN Thorstein (1921), Les ingénieurs et le système de prix, dans Thorstein Veblen, Les ingénieurs et la capitalisme, Paris, Gordon \& Breach - Publications Gramma, , 1971, pp. 1-104

WEINSTEIN Olivier (1997), Modes de coordination interfirmes et modèles d'innovation, dans C.PALLOIX \& Y.RIZOPOULOS (1997)

WILLIAMSON O.E. (1985), Les institutions de l'économie, Paris, InterEditions, 1994, 404 pages

WILLIAMSON O.E. (1996), The mechanisms of governance, NewYork/Oxford, Oxford University Press, 422 pages 